saline-immersion therapeutic endoscopy (SITE) facilitated ESD with a ball-tipped, needle-type ESD knife (Flush Knife-BT $(1.5 \mathrm{~mm})$, Fujifilm, Tokyo, Japan). The resection site was clipped prophylactically and the lipoma was retrieved with a Roth $^{\oplus}$ net (Steris, Mentor, OH, USA) (Fig. 3-4). No immediate, early or late adverse events occurred. Histopathology confirmed complete resection of an ileal lipoma.

Conclusions Our case demonstrates the usefulness of this technique, particularly when combined with the safe and stable endotherapeutic platform afforded by DBE and the buoyancy facilitated by SITE. To the best of our knowledge this is only the 3rd reported case of ESD for a TI lipoma and the only case to date combining the use of SITE-ESD and DBE.

\section{PTU-65 CYANOACRYLATE-INJECTION ENDOTHERAPY FACILITATED BY DOUBLE-BALLOON ENTEROSCOPY: ENDOSCOPIC MANAGEMENT OF ANASTOMOTIC VARICES BLEEDING}

${ }^{1}$ Nikolaos Lazaridis*, ${ }^{1}$ Alberto Murino, ${ }^{1}$ Alexandros Skamnelos, ${ }^{1}$ Rocio Chacchi Cahuin

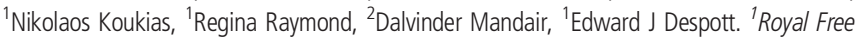
Unit for Endoscopy, The Royal Free Hospital, University College London (UCL) Institute for Liver and Digestive Health, London, UK; ${ }^{2}$ Neuroendocrine Tumour Unit, Centre for Gastroenterology, ENETS Centre of Excellence, The Royal Free Hospital and University College London (UCL) Institute for Liver and Digestive Health, London, UK

\subsection{6/gutjnl-2021-BSG.138}

Introduction Small bowel varices are an uncommon cause of severe gastrointestinal bleeding. There is no consensus regarding the endoscopic management of these patients. Cyanoacrylate injection endotherapy has been described as a therapeutic alternative in some cases.

Methods A 65-year-old man with a metastatic mid-gut neuroendocrine tumour warranting extensive small-bowel resection presented with life-threatening mid-gut bleeding. A CT enterography (CTE) demonstrated jejunal anastomotic varices, with superior mesenteric vein thrombosis. Without a radiological option for treatment, anterograde double-balloon enteroscopy (DBE) was planned to facilitate cyanoacrylate-injection endotherapy (CIE).

Results During DBE 2 nests of bleeding varices were identified at the anastomosis. DBE CIE (diluted in lipiodol) was successful performed through a wide-bore injection-needle. No immediate or delayed adverse events were reported. A subsequent CTE confirmed successful embolisation of the mid-gut varices. The patient has not bled further during 6-months of followup.

Conclusions DBE facilitated CIE of mid-gut varices is effective and safe in carefully selected cases.

\section{PTU-66 MANAGEMENT OF SMALL-BOWEL POLYPS IN PATIENTS WITH PEUTZ-JEGHERS SYNDROME: ISCHAEMIC POLYPECTOMY ASSISTED BY DOUBLE-BALLOON ENTEROSCOPY}

\footnotetext{
${ }^{1}$ Nikolaos Lazaridis, ${ }^{1}$ Alexandros Skamnelos, ${ }^{1}$ Alberto Murino, ${ }^{1,2}$ Erasmia Vlachou, ${ }^{1}$ Nikolaos Koukias, ${ }^{1}$ Rocio Chacchi Cahuin*, 'Sarah Walton, ${ }^{1}$ Regina Raymond, ${ }^{1}$ Edward J Despott. 'Royal Free Unit for Endoscopy, The Royal Free Hospital, University College London (UCL) Institute for Liver and Digestive Health, London, UK; ${ }^{2}$ Department of Gastroenterology and Endoscopy, NIMTS Army Veterans Hospital, Athens, Greece
}

10.1136/gutjnl-2021-BSG.139
Introduction Ischaemic polypectomy with detachable-snares or endoclips at double-balloon enteroscopy (DBE) is emerging as a promising technique for the minimally-invasive management of small-bowel polyps in patients with Peutz-Jeghers syndrome (PJS).

Methods The aim of our study was to review our experience of ischaemic polypectomies in patients with PJS from July 2017 to November 2020. Demographic, endoscopic, histopathological data were analysed.

Results In this case series, 46 polyps were treated during 9 therapeutic DBEs (6 anterograde and 3 retrograde) performed in 4 patients ( 3 male and 1 female). Seven polyps $>20 \mathrm{~mm}$ in size required loop ligation with a detachable-snare. The majority of the small bowel polyps (39 lesions) were $<20 \mathrm{~mm}$ and were treated successfully with the double-clipping technique. No immediate or late adverse events occurred. All patients remained asymptomatic during follow-up (mean 19; range 148 months). None required subsequent surgery.

\begin{tabular}{lllll} 
Abstract PTU-66 Tabel 1 \\
$\begin{array}{llll}\text { Age/ } \\
\text { Gender }\end{array}$ & $\begin{array}{l}\text { Number of procedures } \\
\text { (antero/retro) }\end{array}$ & $\begin{array}{l}\text { Total } \\
\text { polyps }\end{array}$ & Clipping & $\begin{array}{l}\text { Detachable snare for } \\
\text { polyps }>20 \mathrm{~mm}\end{array}$ \\
\hline 50/M & $2(1 / 1)$ & 17 & 17 & - \\
$56 / \mathrm{M}$ & $1(1 /-)$ & 14 & 14 & - \\
29/F & $3(1 / 2)$ & 7 & 4 & 3 \\
29/M & $3(3 /-)$ & 8 & 4 & 4 \\
\hline
\end{tabular}

Conclusions Ischaemic polypectomy facilitated by DBE appears to be a safe and effective endotherapeutic option for endotherapy of small-bowel polyps in patients with PJS.

\section{IBD}

\section{PMO-1 IDENTIFICATION OF A NOVEL COLONIC MACROPHAGE SIGNATURE IN A SUBSET OF CROHN'S DISEASE PATIENTS}

${ }^{1}$ Hannah Baer*, ${ }^{2}$ Elizabeth McDonald, ${ }^{3}$ Muhammad I Khan, ${ }^{4}$ Umer I Zeeshan, ${ }^{1}$ John Cole ${ }^{5}$ Meera Ramanujam, ${ }^{1}$ Robert BJ Nibbs, ${ }^{1}$ Simon WF Milling. 'Institute of Infection, Inflammation and Immunity - University of Glasgow, Glasgow, UK; ${ }^{2}$ Medical Research Institute - QIMR Berghofer, Brisbane, Australia; ${ }^{3}$ Gastroenterology Unit - Glasgow Royal Infirmary, Glasgow, UK; ${ }^{4}$ School of Engineering - University of Glasgow, Glasgow, UK; ${ }^{5}$ Boehringer Ingelheim Pharmaceuticals Inc, Immunology and Respiratory Diseases Research, Ridgefield, USA

\subsection{6/gutjnl-2021-BSG.140}

Introduction Inflammatory bowel diseases (IBD) are conditions characterised by chronic gastrointestinal inflammation resulting from abnormal immune responses against gut microbiota. Crohn's disease (CD) is one of the two common forms of IBD which can manifest as inflammation anywhere along the gastrointestinal tract, causing potentially life-threatening complications. A wide range of immunomodulatory therapies are available for IBD treatment. However response rates are often suboptimal and many patients still require resective surgery. Therapeutic outcomes could be significantly improved by identifying biomarkers that predict treatment responses in CD patients and by developing a deeper understanding of the different molecular mechanisms that drive inflammation. 